\title{
CADASIL. Estudio neuropatológico de un caso
}

\author{
Beatriz San Millán Tejado $1,6^{*}$, Begoña Iglesias Rodríguez ${ }^{2,6^{*}}$, Manuel Seijo Martínez ${ }^{3,7}$, \\ Susana Teijeira Bautista ${ }^{4,6}$ y Carmen Navarro Fernández-Balbuena ${ }^{5,6}$ \\ ${ }^{1}$ Neuropatóloga. \\ ${ }^{2}$ Patóloga. \\ 3 Neurólogo. \\ ${ }^{4}$ Doctora en Biología. \\ ${ }^{5}$ Neuropatóloga y doctora en Medicina. \\ كServicio de Anatomía Patológica. Neuropatología-Banco de Tejidos Neurológicos de Vigo. \\ Complejo Hospitalario Universitario de Vigo (Meixoeiro). \\ ${ }^{7}$ Servicio de Neurología. Hospital do Salnés. Pontevedra. \\ *Beatriz San Millán y Begoña Iglesias han contribuido en igual medida en la elaboración de este artículo.
}

\section{Resumen}

Fundamento: la arteriopatía cerebral autosómica dominante con infartos subcorticales y leucoencefalopatía (CADASIL) es la primera causa hereditaria de infartos cerebrales y demencia vascular en adultos. Objetivo: presentar los hallazgos histopatológicos y ultraestructurales en una paciente con CADASIL en el momento del diagnóstico y en el estudio post mortem del sistema nervioso central, 10 años después. Pacientes y métodos: se estudió a una paciente con migraña precedida de aura, ictus y demencia con historia familiar en varias generaciones. Se realizaron pruebas de neuroimagen, biopsias de piel y músculo esquelético, además de estudio molecular de Notch 3. Se examinaron muestras post mortem del encéfalo, piel y músculo esquelético. Resultados: la resonancia magnética y la tomografía computarizada mostraron una extensa leucoaraiosis. El examen ultraestructural de las biopsias demostró la presencia de depósitos granulares electrondensos en las paredes vasculares, característicos de CADASIL. Se detectó una mutación en el gen Notch 3. El estudio neuropatológico post mortem mostró infartos múltiples, desmielinización y alteraciones de las paredes vasculares, con depósitos granulares densos. Conclusiones: en el examen neuropatológico se observaron los hallazgos de CADASIL evolucionado. Las muestras de piel y músculo obtenidas en el estudio post mortem demostraron la progresión de las lesiones vasculares. La presencia de ictus tempranos en individuos con historia familiar positiva debe plantear el diagnóstico de CADASIL.

(Alzheimer. Real Invest Demenc. 2011 ;48:5-12)

Palabras clave: CADASIL, Notch 3, demencia vascular, ultraestructura, GOM.

\section{Abstract}

Background: cerebral autosomal dominant arteriopathy with subcortical infarcts and leukoencephalopathy (CADASIL) is the most common inherited cause of stroke and vascular dementia in adults. Objective: to report the histopathological and ultrastructural findings in a patient with CADASIL at the time of diagnosis and in the post mortem study of the central nervous system, 10 years later. Patients and methods: we studied a patient with migraine with aura and a family history of migraine, stroke and dementia. Imaging tests, skin and muscle biopsies and molecular studies of Notch 3 were performed. In the post mortem examination, representative regions of the brain, muscle and skin samples were studied. Results: striking leukoaraiosis was found on MRI. Electrondense granular deposits characteristic of CADASIL were identified in the vascular walls in the biopsy samples. A mutation was detected in Notch 3 gene. Post mortem examination showed multiple brain infarcts, demyelination and vascular wall thickening, with striking granular deposits. Conclusions: neuropathological examination showed the characteristic findings of evolved CADASIL in the brain, skin and skeletal muscle vessels. The presence of early strokes in patients with family history should suggest the diagnosis of CADASIL

(Alzheimer. Real Invest Demenc. 2011;48:5-12)

Keywords: CADASIL, Notch 3, vascular dementia, ultrastructure, GOM 


\section{Introducción}

La arteriopatía cerebral autosómica dominante con infartos subcorticales y leucoencefalopatía (CADASIL, Cerebral Autosomal Dominant Arteriopathy with Subcortical Infarcts and Leukoencephalopathy) es una angiopatía hereditaria sistémica muy infrecuente, que se manifiesta en la edad adulta como cefalea migrañosa con aura y accidentes cerebrales isquémicos. Su curso clínico es lentamente progresivo, con la aparición de síntomas neuropsiquiátricos y demencia en los estadios más avanzados. La mayoría de los pacientes tiene familiares de primer grado afectos, según un patrón de herencia autosómica dominante, aunque se han descrito casos esporádicos ${ }^{1}$.

Las manifestaciones clínicas de CADASIL son exclusivamente neurológicas, a pesar de que los vasos extracerebrales de distintas localizaciones presentan la misma patología ${ }^{2}$. La resonancia magnética $(\mathrm{RM})$ cerebral es la técnica de imagen más útil para demostrar las alteraciones de CADASIL. En la mayoría de los pacientes se encuentran lesiones hiperintensas con T2 y FLAIR de localización en sustancia blanca, cápsula externa, ganglios basales y protuberancia ${ }^{3}$ desde edades tempranas, que con la tomografía computarizada (TC) y la resonancia magnética nuclear (RMN) T1 son hipointensas y caracterizan a los infartos lacunares ${ }^{4,5}$.

El gen responsable, Notch 3, identificado en 1996 en 19p13.2-p13.16, codifica un receptor transmembrana fundamental para el desarrollo tímico, neurológico y vascular ${ }^{7,8}$, así como para la remodelación vascular postlesional ${ }^{9,10}$. Se han descrito más de 100 mutaciones, distribuidas en 20 de sus 33 exones y más de 500 familias afectadas en todo el mundo ${ }^{11}$.

Los estudios neuropatológicos post mortem muestran las alteraciones típicas de las angiopatías cerebrales crónicas de vaso pequeño. Microscópicamente se encuentra rarefacción de la sustancia blanca de pre-

Recibido para su publicación: 31 de agosto de 2010. Aceptado para su publicación: 15 de septiembre de 2010. Correspondencia: B. San Millán Tejado

E-mail: beatriz.san.millan.tejado@sergas.es dominio periventricular, infartos lacunares en sustancia blanca y ganglios basales, además de estado criboso $^{2}$, con desmielinización irregular, dilatación de los espacios perivasculares y gliosis astrocitaria leve. Esta angiopatía afecta de manera selectiva a las arterias pequeñas (de 100 a $400 \mu \mathrm{m}$ de diámetro) y a los capilares, tanto en el sistema nervioso central (SNC) como a nivel sistémico ${ }^{2}$. Las paredes vasculares muestran depósitos granulares en la capa media, fibrosis y oclusión de las luces vasculares ${ }^{12}$.

El hallazgo ultraestructural patognomónico de CADASIL son los depósitos granulares osmiofílicos (GOM, del inglés granular osmiophilic material) en las paredes vasculares. Se localizan alrededor de las células endoteliales, musculares lisas y pericitos en los vasos del SNC y, a nivel sistémico, en los vasos del músculo esquelético, nervio periférico, piel, intestino delgado y grueso, hígado, riñón y corazón ${ }^{13,14}$. Esta observación ha permitido la utilización de la biopsia cutánea como método fiable y mínimamente invasivo para el diagnóstico ${ }^{2,15}$, esencial para la aplicación de medidas preventivas y paliativas, eventual detección de familiares afectos y realización de consejo genético. Actualmente no existe una terapia efectiva, sino que se limita al tratamiento sintomático y de las complicaciones agudas.

Describimos los hallazgos histopatológicos y ultraestructurales en una paciente diagnosticada de CADASIL por biopsia de piel y músculo esquelético 10 años antes de su fallecimiento, así como los del SNC, piel y músculo realizados post mortem.

\section{Paciente}

Se trata de una mujer de 69 años con cefalea episódica de dos décadas de evolución, precedida por fenómenos de aura consistentes en debilidad del miembro superior derecho, disfasia, alteraciones visuales o parestesias en hemicara y brazo derechos. Tras el aura se instauraba una cefalea intensa frontal y hemicraneal derecha, pulsátil, con fotofobia y sonofobia, malestar general, náuseas y vómitos. Los episodios ocurrían entre 1 y 4 veces al mes, con una duración de 24 horas y cedían con paracetamol o antiinflamatorios no esteroideos. 
La paciente refería a varios familiares fallecidos con demencia, alteraciones neuropsiquiátricas y accidentes cerebrovasculares (ACV), además de varios familiares vivos con sintomatología similar, a lo largo de varias generaciones. La exploración neurológica inicial fue normal, así como la analítica general, bioquímica, y extensos estudios serológicos y de coagulación.

La RMN y la TC mostraron amplias lesiones periventriculares con características de leucoaraiosis grado IV (Van Swieten), que se extendían hasta la región subcortical (fig. 1). Los ganglios basales presentaban lesiones de aspecto isquémico crónico. Ante la sospecha clínica de enfermedad mitocondrial, se obtuvieron biopsias de piel y músculo esquelético, y se estableció, tras su estudio, el diagnóstico de CADASIL.

Tres años después se instauraron alteraciones de la memoria reciente, enlentecimiento mental y motor progresivos, que evolucionaron a un cuadro de demencia claramente establecida seis años después. El tratamiento con donepecilo no tuvo ningún efecto
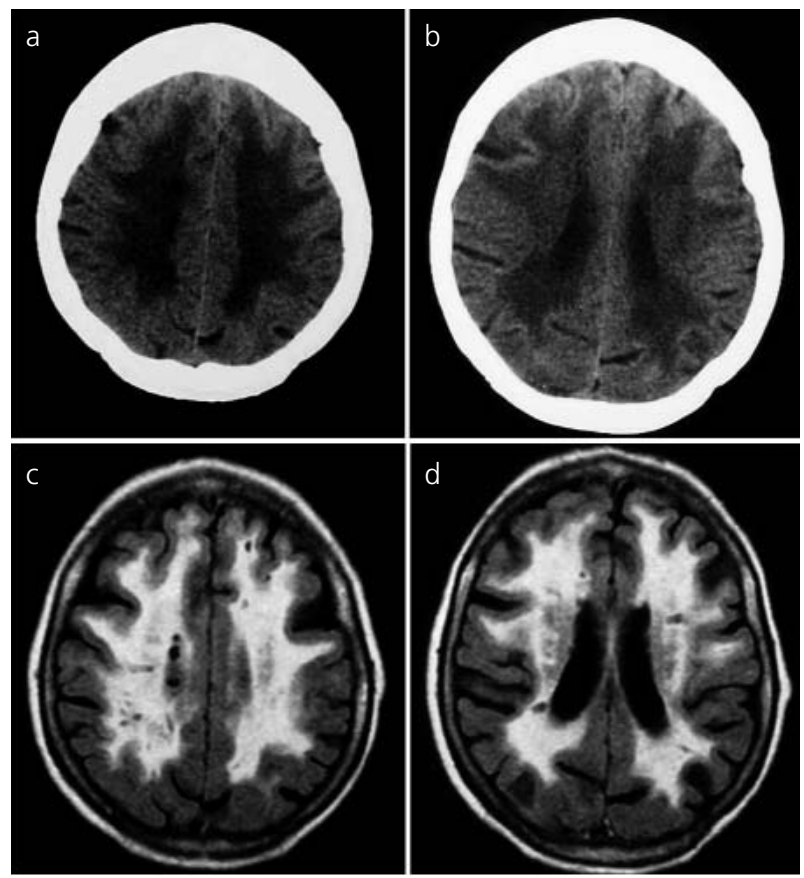

Figura 1. Tomografía computarizada (TC) y resonancia magnética nuclear (RMN) cerebrales: leucoaraiosis extensa periventricular con extensión subcortical. Hipodensidad con TC (a-b) e hiperintensidad en RMN (c-d). Dilatación de los ventrículos laterales y estado criboso $(c-d)$. significativo. La paciente sufrió varios episodios de neumonía y falleció por sepsis de origen respiratorio, 10 años después del diagnóstico.

Se realizó necropsia limitada al SNC y se examinaron muestras post mortem de músculo esquelético y piel.

\section{Resultados}

El examen histológico de las biopsias de piel y músculo no mostró hallazgos patológicos. No se observó proliferación mitocondrial y el estudio bioquímico de la cadena respiratoria mitocondrial (Dr. MA Martín, hospital 12 de Octubre, Madrid) fue normal. El examen ultraestructural reveló depósitos electrondensos granulares no limitados por membrana, de 10-15 nm de diámetro, en las paredes vasculares (fig. 2 a y 2 b), que formaban agregados adyacentes a las células endoteliales, entre las células musculares lisas o situados en indentaciones de las células o en el espesor de la membrana basal (fig. 2a y 2c). Ante estos hallazgos se realizó el diagnóstico de CADASIL, que se confirmó con el estudio genético de Notch 3.

En el examen post mortem, el hemisferio cerebral derecho fue incluido en el banco de tejidos neurológicos de Vigo. Se seleccionaron múltiples áreas representativas del hemisferio izquierdo, que se procesaron con tinciones de hematoxilina-eosina (HE), ácido peryódico de Schiff (PAS, del inglés periodic acid-Schiff), tricrómico de Masson, orceína e inmunohistoquímica para proteína glial fibrilar ácida (GFAP, del inglés glial fibrillary acidic protein), beta amiloide, alfa-sinucleína, proteína tau y ubiquitina.

El encéfalo pesaba $1.020 \mathrm{~g}$ y mostraba diversos infartos cavitados (fig. 3a), además de un extenso infarto antiguo en el territorio de la arteria cerebral posterior izquierda (fig. 3b). Las secciones coronales mostraron múltiples infartos córtico-subcorticales, rarefacción periventricular y en centro semioval, además de atrofia marcada del cuerpo calloso (fig. 3c). La sustancia blanca y los ganglios basales presentaban estado criboso y lacunar característico. En los vasos se observaron abundantes placas ateromatosas (fig. 3b), con gran reducción de sus luces (fig. 3c). 

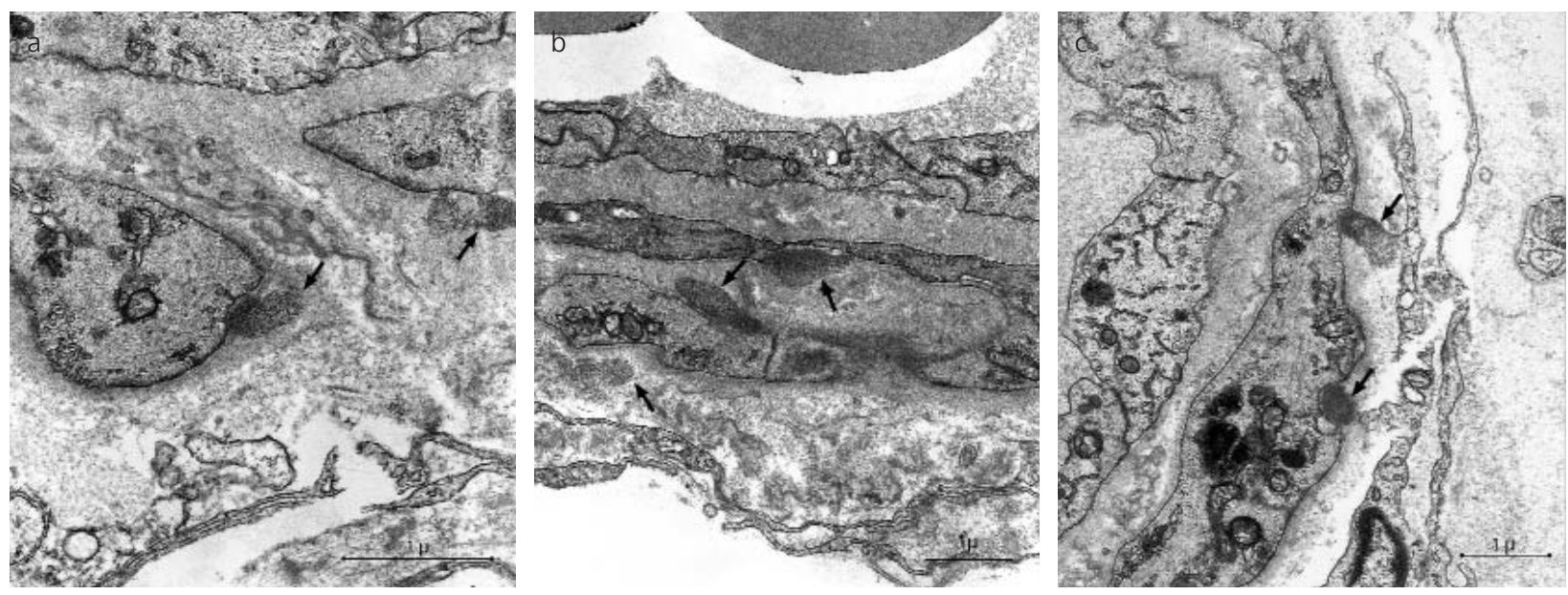

Figura 2. Hallazgos ultraestructurales en las biopsias cutánea (a) y muscular (b y c). Depósitos vasculares de material granular osmiofílico extracelular y no rodeado de membrana, en las indentaciones de las células musculares lisas y adyacentes a las células endoteliales (magnificaciones originales a, b, c: $8.900 \mathrm{X}$ ).
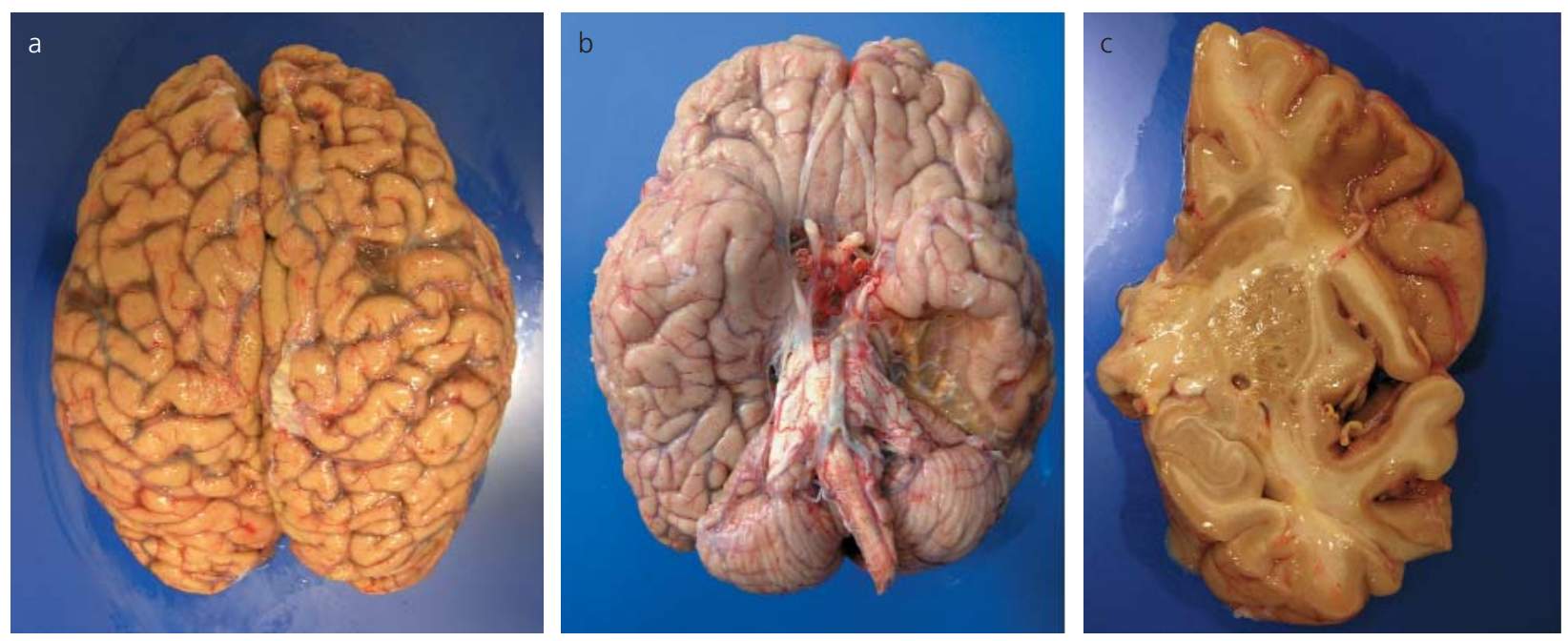

Figura 3. Hallazgos macroscópicos en el sistema nervioso central. a) Infartos frontoparietales bilaterales en la convexidad cerebral, y atrofia cortical frontal. b) Visión basal: infarto temporooccipital izquierdo antiguo en el territorio de la arteria cerebral posterior. Ateromatosis severa. c) Sección coronal del hemisferio izquierdo: infarto corticosubcortical en circunvolución frontal media. Estado criboso en putamen, pallidum y claustro. Nótese el gran adelgazamiento del cuerpo calloso.

El examen microscópico mostró múltiples infartos en la sustancia blanca de todos los lóbulos, ganglios basales, tálamo, tronco encefálico y cerebelo. El estadio y el diámetro de los infartos eran variables, con lesiones antiguas quistificadas, infiltrados de macrófagos espumosos, y proliferación microvascular y astroglial intensa en el parénquima circundante. Los espacios perivasculares de Virchow-Robin (VRS, del inglés Vi-
rchow-Robin space) estaban marcadamente dilatados y revestidos por invaginaciones de la piamadre (fig. 4a-b), correspondientes al estado criboso. La sustancia blanca presentaba además múltiples áreas de desmielinización, perivascular y periventricular.

Las paredes de los vasos de pequeño y mediano calibre intraparenquimatosos y leptomeníngeos se encontraban engrosadas (fig. 4c), con desdoblamiento de la 


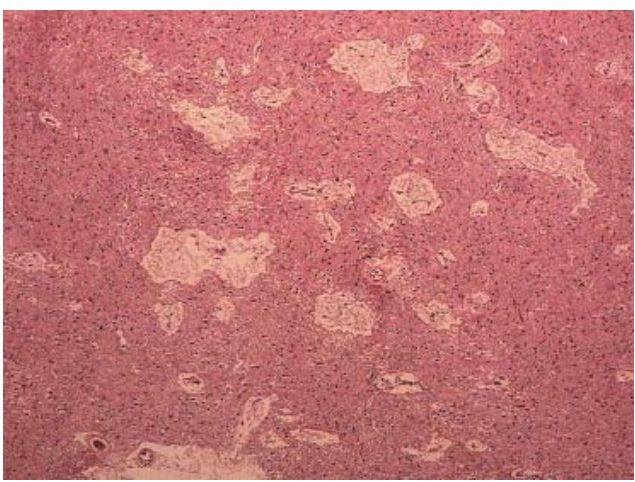

b
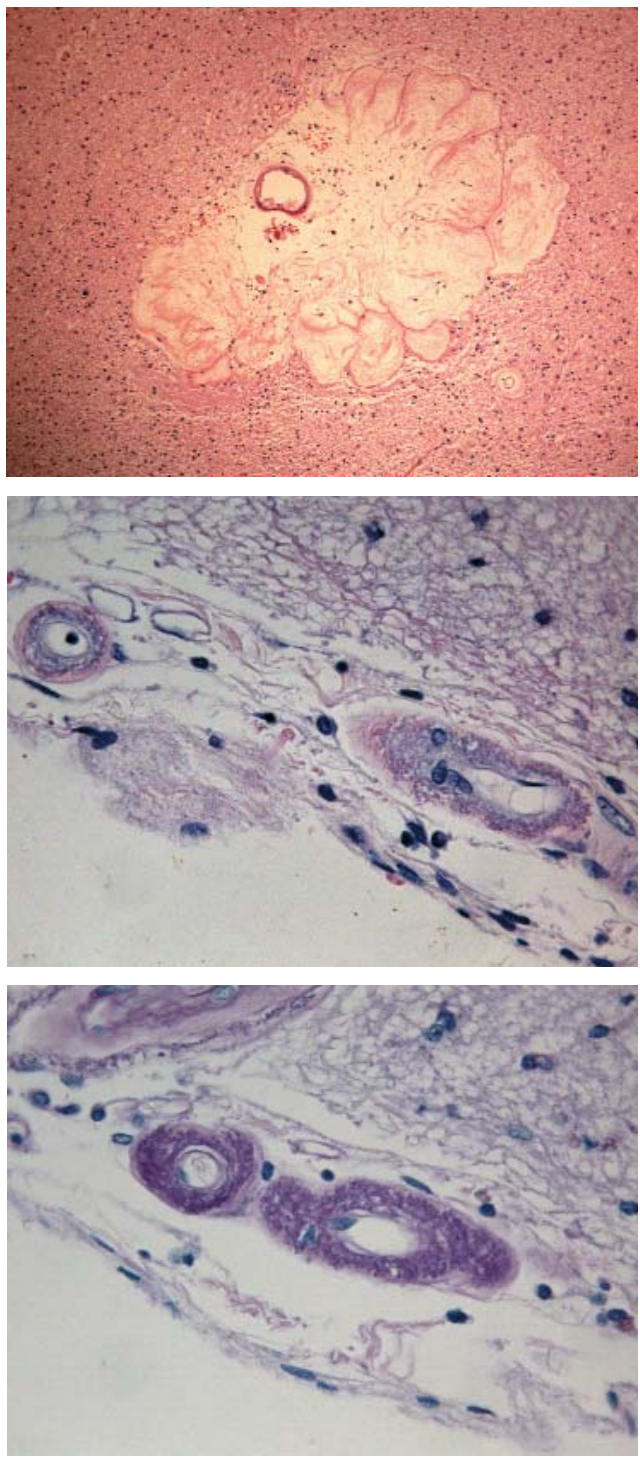

Figura 4. Alteraciones histopatológicas cerebrales en CADASIL. a y b) Dilatación de los espacios perivasculares característica del «estado criboso» en el estriado (a: HE 5X, b: HE10X). c y d) Engrosamiento de las paredes vasculares y depósitos granulares, basófilos con HE (c) y PAS positivos (d) [c: HE 63X; d: PAS 63X]. lámina elástica interna y desestructuración de la capa media arteriolar, y mostraban depósitos granulares densos, basófilos con HE (fig. 4c) y PAS positivos (fig. 4d). No se observaron placas seniles o neuríticas, figuras de degeneración neurofibrilar ni angiopatía amiloidea.

El examen ultraestructural evidenció los característicos GOM (fig. 5a-b), con un diámetro medio de 10$15 \mathrm{~nm}$, formando agregados de hasta $1 \mu \mathrm{m}$ adyacentes a las células musculares lisas y a los pericitos de las arterias y arteriolas, o junto a las células endoteliales en los capilares. Los depósitos eran extracelulares y separaban anormalmente las células de las paredes vasculares, provocando un engrosamiento de las mismas. Ocasionalmente se encontraron cambios degenerativos y engrosamiento de la membrana basal (fig. 5d).

En las muestras post mortem de músculo y piel (fig. 5c-d) se objetivó una progresión llamativa de las lesiones encontradas 10 años antes, con aumento de los depósitos osmiófilos en las paredes vasculares y del número de vasos afectados.

\section{Discusión}

La arteriopatía cerebral autosómica dominante con infartos subcorticales y leucoencefalopatía afecta de forma similar a ambos sexos, con una gran variabilidad inter e intrafamiliar en la edad de inicio. Los afectados presentan ataques de migraña que suelen aparecer en la tercera década de la vida y episodios ictales entre los 28 y los 60 años de edad ${ }^{16}$. Se ha descrito un inicio más precoz en mujeres, en ocasiones desencadenado por la gestación ${ }^{17}$. La paciente estudiada presentó una edad de inicio relativamente tardía, en la quinta década de la vida, con cefaleas episódicas precedidas de auras complejas y posteriormente alteraciones cognitivas, demencia y ataxia. Refería familiares de ambos sexos con una clínica similar a lo largo de cinco generaciones, característica del patrón de herencia autosómica dominante.

El diagnóstico de CADASIL requiere la identificación de una mutación en Notch 3, o la demostración de depósitos GOM por microscopía electrónica en las paredes vasculares con una especificidad, en la biop- 
a

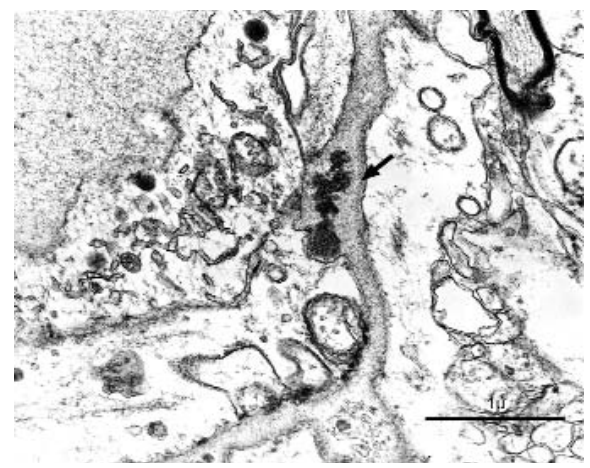

b

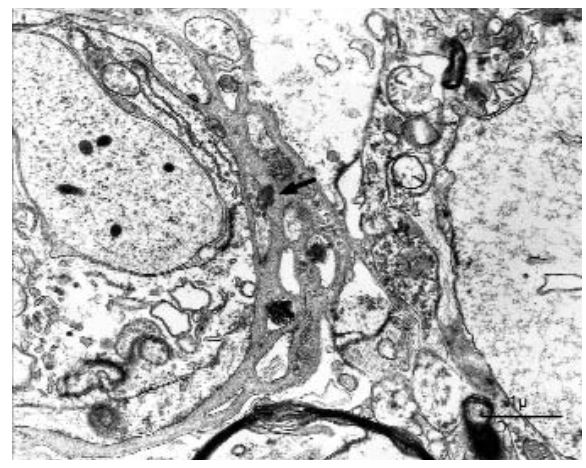

C

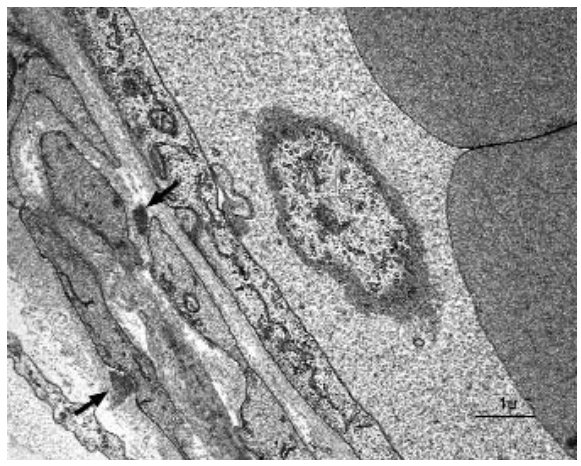

d

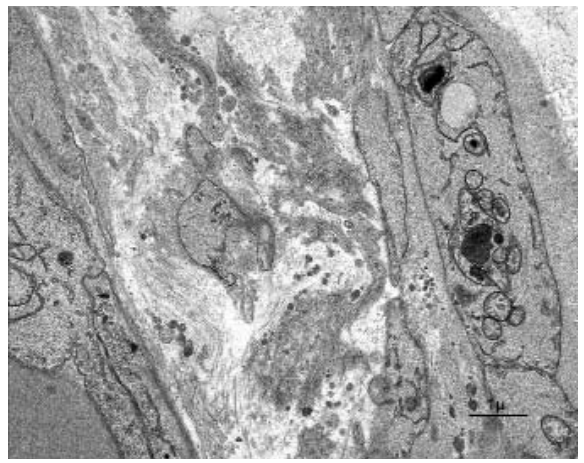

Figura 5. Hallazgos ultraestructurales post mortem en vasos del sistema nervioso central (a-b) y en la piel (c-d). Presencia de material granular osmiofílico en las paredes vasculares (flechas $a, b$ y c) y material filamentoso degenerativo entre las células musculares lisas, con engrosamiento de la membrana basal vascular (d). (Magnificaciones originales a: 15.500X; b: 8.900X; c y d: 10.500X.) sia cutánea, que se aproxima al $100 \%$ y una sensibilidad del $45-96 \%{ }^{18}$. En la paciente que referimos aquí, el diagnóstico se estableció por el hallazgo de GOM en las biopsias de piel y de músculo, y posteriormente se halló una mutación en el gen Notch 3.

Los GOM, cuya composición bioquímica se desconoce, constituyen el hallazgo ultraestructural patognomónico de CADASIL, aunque la intensidad y la cantidad de los depósitos son muy variables, y pueden ser sólo focales ${ }^{19}$, por lo que es necesario el examen minucioso de un número elevado de vasos. Su localización preferente en la proximidad de las células musculares lisas y los pericitos de las paredes vasculares es interesante, ya que estas células son las únicas células maduras que mantienen activa la expresión del receptor Notch 3. En la membrana plasmática celular se ha demostrado, mediante estudios inmunohistoquímicos, la presencia de depósitos del dominio extracelular de Notch 3, adyacentes a los GOM, pero independientes de éstos ${ }^{20,21}$. Se desconoce la relación entre la formación de GOM y el acúmulo de Notch 3, así como su papel patogénico en la lesión parietal vascular.

Por otra parte, se han demostrado otras alteraciones en los vasos, como engrosamiento de la membrana basal, degeneración de las células endoteliales y de las células musculares lisas de la capa media ${ }^{22}$ y fibrosis de la adventicia ${ }^{23}$. Ello da lugar al aumento del espacio subendotelial e intercelular, con el consiguiente incremento del espesor de las paredes vasculares y oclusión progresiva de sus luces ${ }^{12}$. Esta patología era muy manifiesta en los vasos perforantes de pequeño calibre del SNC (fig. 4), con acúmulos granulares basófilos PAS positivos en sus paredes que correspondían a depósitos granulares osmiofílicos a nivel ultraestructural.

Se ha demostrado la afectación de los vasos extracerebrales de distintas localizaciones, como músculo esquelético y cardíaco, nervio periférico, piel, intestino delgado y grueso, hígado y riñón ${ }^{13,14}$; por lo tanto, se ha considerado que CADASIL es una enfermedad sistémica. Sin embargo, por razones que se desconocen, sus manifestaciones son exclusivamente neurológicas, y algunos autores señalan las características anatómicas particulares de la barrera hematoencefálica como factor contribuyente a este hecho 2,12,24. 
Otro hallazgo histológico frecuente es la dilatación importante de los VRS, que forman grandes cavidades quísticas perivasculares revestidas por una única capa de células aplanadas, las cuales constituyen una invaginación de la piamadre ${ }^{25-29}$. En CADASIL, estas lesiones se encuentran principalmente en los ganglios basales y en la sustancia blanca temporal. El estado criboso se acentúa con la edad de los pacientes y con la progresión de las lesiones vasculares ${ }^{25,30}$. Igualmente se sugiere que la dilatación de los VRS puede estar relacionada con la disminución del volumen del parénquima cerebral por las lesiones progresivas de desmielinización y pérdida axonal ${ }^{25}$.

En resumen, los hallazgos clínicos y neuropatológicos de la paciente que describimos son los característicos de CADASIL de larga evolución; se resalta que la comparación de las muestras de piel y músculo obtenidas en el momento del diagnóstico y en el examen post mortem, 10 años más tarde, pusieron en evidencia la progresión de las lesiones histopatológicas y ultraestructurales, en ausencia de manifestaciones clínicas extracerebrales.

El diagnóstico de CADASIL debe plantearse ante la presencia de ACV en edades tempranas, especialmente en pacientes con antecedentes familiares de episodios isquémicos, migraña o demencia subcortical. El examen ultraestructural de la biopsia cutánea o muscular es de gran utilidad diagnóstica, aunque dado el carácter focal de la afectación vascular, es necesario el estudio minucioso de la biopsia por personal experimentado. La ausencia de depósitos en una biopsia no excluye el diagnóstico y debe realizarse un estudio genético de los casos altamente sugestivos.

\section{Agradecimientos:}

A Soraya Barrera, por su excelente trabajo técnico. A Tania y Cristina Vázquez, por su ayuda en la edición de este artículo. Este trabajo ha sido subvencionado en parte por el Fondo de Investigación Sanitaria (PI07/1257, PI07/90043 y RD09/0076/ 00011 ) y por la Xunta de Galicia (INCITE07PXI905221ES, PS08/38, 09CSA051905PR, REGENPSI 2009/019). Beatriz San Millán está contratada por el «programa Río Hortega» del Instituto de Salud Carlos III (Exp. M07/00135).

\section{Bibliografía}

1. Joutel A, Dodick DD, Parisi JE, Cecillon M, TournierLasserve E, Bousser MG. De novo mutation in the Notch 3 gene causing CADASIL. Ann. Neurol. 2000 Mar; 47(3):388-91.

2. Ruchoux MM, Maurage CA. CADASIL: Cerebral autosomal dominant arteriopathy with subcortical infarcts and leukoencephalopathy. J.Neuropathol.Exp.Neurol. 1997 Sep;56(9):947-64.

3. Andre C. CADASIL: pathogenesis, clinical and radiological findings and treatment. Arq. Neuropsiquiatr. 2010 Apr; 68(2):287-99.

4. Chabriat H, Vahedi K, Iba-Zizen MT, Joutel A, Nibbio A, Nagy TG, et al. Clinical spectrum of CADASIL: a study of 7 families. Cerebral autosomal dominant arteriopathy with subcortical infarcts and leukoencephalopathy. Lancet. 1995 Oct 7;346(8980):934-9.

5. Herve D, Mangin JF, Molko N, Bousser MG, Chabriat H. Shape and volume of lacunar infarcts: a 3D MRI study in cerebral autosomal dominant arteriopathy with subcortical infarcts and leukoencephalopathy. Stroke. 2005 Nov; 36(11):2384-8.

6. Joutel A, Corpechot C, Ducros A, Vahedi K, Chabriat H, Mouton P, et al. Notch 3 mutations in CADASIL, a hereditary adult-onset condition causing stroke and dementia. Nature. 1996 Oct 24;383(6602):707-10.

7. Arboleda-Velasquez JF, Zhou Z, Shin HK, Louvi A, Kim $\mathrm{HH}$, Savitz SI, et al. Linking Notch signaling to ischemic stroke. Proc. Natl. Acad. Sci. U.S.A. 2008 Mar 25; 105(12):4856-61.

8. Domenga V, Fardoux P, Lacombe P, Monet M, Maciazek J, Krebs LT, et al. Notch 3 is required for arterial identity and maturation of vascular smooth muscle cells. Genes Dev. 2004 Nov 15;18(22):2730-5.

9. Wang W, Prince CZ, Mou Y, Pollman MJ. Notch 3 signaling in vascular smooth muscle cells induces c-FLIP expression via ERK/MAPK activation. Resistance to Fas ligand-induced apoptosis. J.Biol.Chem. 2002 Jun 14; 277(24):21723-9.

10. Morrow D, Sweeney C, Birney YA, Cummins PM, Walls D, Redmond EM, et al. Cyclic strain inhibits Notch receptor signaling in vascular smooth muscle cells in vitro. Circ. Res. 2005 Mar 18;96(5):567-75.

11. Chabriat H, Joutel A, Dichgans M, Tournier-Lasserve E, Bousser MG. Cadasil. Lancet Neurol. 2009 Jul;8(7):64353.

12. Miao Q, Paloneva T, Tuominen S, Poyhonen M, Tuisku S, Viitanen M, et al. Fibrosis and stenosis of the long penetrating cerebral arteries: the cause of the white matter pathology in cerebral autosomal dominant arteriopathy with subcortical infarcts and leukoencephalopathy. Brain Pathol. 2004 Oct;14(4):358-64. 
13. Ruchoux MM, Guerouaou D, Vandenhaute B, Pruvo JP, Vermersch P, Leys D. Systemic vascular smooth muscle cell impairment in cerebral autosomal dominant arteriopathy with subcortical infarcts and leukoencephalopathy. Acta Neuropathol. 1995;89(6):500-12.

14. Bergmann M, Ebke M, Yuan Y, Bruck W, Mugler M, Schwendemann G. Cerebral autosomal dominant arteriopathy with subcortical infarcts and leukoencephalopathy (CADASIL): a morphological study of a German family. Acta Neuropathol. 1996 Oct;92(4):341-50.

15. Kalimo H, Ruchoux MM, Viitanen M, Kalaria RN. CADASIL: a common form of hereditary arteriopathy causing brain infarcts and dementia. Brain Pathol. 2002 Jul;12(3):371-84.

16. Desmond DW, Moroney JT, Lynch T, Chan S, Chin SS, Mohr JP. The natural history of CADASIL: a pooled analysis of previously published cases. Stroke. 1999 Jun; 30(6):1230-3.

17. Roine S, Poyhonen M, Timonen S, Tuisku S, Marttila R, Sulkava R, et al. Neurologic symptoms are common during gestation and puerperium in CADASIL. Neurology. 2005 Apr 26;64(8):1441-3.

18. Markus HS, Martin RJ, Simpson MA, Dong YB, Ali N, Crosby $\mathrm{AH}$, et al. Diagnostic strategies in CADASIL. Neurology. 2002 Oct 22;59(8):1134-8.

19. Schultz A, Santoianni R, Hewan-Lowe K. Vasculopathic changes of CADASIL can be focal in skin biopsies. Ultrastruct.Pathol. 1999 Jul-Aug;23(4):241-7.

20. Ishiko A, Shimizu A, Nagata E, Takahashi K, Tabira T, Suzuki N. Notch 3 ectodomain is a major component of granular osmiophilic material (GOM) in CADASIL. Acta Neuropathol. 2006 Sep;1 12(3):333-9.

21. Joutel A, Andreux F, Gaulis S, Domenga V, Cecillon M, Battail N, et al. The ectodomain of the Notch 3 receptor accumulates within the cerebrovasculature of CADASIL patients. J.Clin.Invest. 2000 Mar; 105(5):597-605.

22. Lewandowska E, Leszczynska A, Wierzba-Bobrowicz T, Skowronska M, Mierzewska H, Pasennik E, et al. Ultras- tructural picture of blood vessels in muscle and skin biopsy in CADASIL. Folia Neuropathol. 2006;44(4):26573.

23. Okeda R, Arima K, Kawai M. Arterial changes in cerebral autosomal dominant arteriopathy with subcortical infarcts and leukoencephalopathy (CADASIL) in relation to pathogenesis of diffuse myelin loss of cerebral white matter: examination of cerebral medullary arteries by reconstruction of serial sections of an autopsy case. Stroke. 2002 Nov;33(11):2565-9.

24. Dichgans M, Wick M, Gasser T. Cerebrospinal fluid findings in CADASIL. Neurology. 1999 Jul 13;53(1):233.

25. Cumurciuc R, Guichard JP, Reizine D, Gray F, Bousser MG, Chabriat H. Dilation of Virchow-Robin spaces in CADASIL. Eur.J.Neurol. 2006 Feb;13(2):187-90.

26. Benhaiem-Sigaux N, Gray F, Gherardi R, Roucayrol AM, Poirier J. Expanding cerebellar lacunes due to dilatation of the perivascular space associated with Binswanger's subcortical arteriosclerotic encephalopathy. Stroke. 1987 Nov-Dec;18(6):1087-92.

27. Vital C, Julien J. Widespread dilatation of perivascular spaces: a leukoencephalopathy causing dementia. Neurology. 1997 May;48(5):1310-3.

28. Song CJ, Kim JH, Kier EL, Bronen RA. MR imaging and histologic features of subinsular bright spots on T2-weighted MR images: Virchow-Robin spaces of the extreme capsule and insular cortex. Radiology. 2000 Mar;214(3): $671-7$.

29. Machado MA Jr, Matos AS, Goyanna F, Barbosa VA, Vieira LC. Dilatation of Virchow-Robin spaces in patients with migraine. Arq.Neuropsiquiatr. 2001 Jun;59(2-A): 206-9.

30. Poirier J, Barbizet J, Gaston A, Meyrignac C. Thalamic dementia. Expansive lacunae of the thalamo-paramedian mesencephalic area. Hydrocephalus caused by stenosis of the aqueduct of Sylvius. Rev.Neurol.(Paris) 1983;139(5): 349-58. 